\title{
Application of molecular tools for the diagnosis of central nervous system infections
}

\author{
Serena Delbue ${ }^{1}$, Sara Tremolada ${ }^{2}$, and Pasquale Ferrante Tr, $^{1, \varpi}$ \\ 1 Department of Biomedical Sciences and Technologies, University of Milan, Milan, Italy \\ 2 Center for Translational Research, Ospedale San Giuseppe Milanocuore, Via San Vittore 12, 20123 Milan, \\ Italy
}

\begin{abstract}
Many infectious agents can cause central nervous system (CNS) diseases in humans. Since microbial agents infecting CNS are numerous and have different features, conventional laboratory tests may not be sensitive enough to identify and characterise viruses and bacteria in human biological specimens. Thus, the need to define methods for the diagnosis of infectious neurological diseases, such as progressive multifocal leukoencephalopathy (PML), is urgent, in order to improve the outcome of the diseases with rapid and accurate detection of the pathogens.
\end{abstract}

\section{Keywords}

Central nervous system; Infections; Virus; DNA amplification; PCR

Laboratory diagnosis of an infectious cause of disease is a complex process that starts with identification of the causative agent in an appropriate clinical specimen. However, a significantly high number of pathogens remains unrecognised, resulting in high rates of mortality. Critical and timely intervention for infectious diseases would rely on rapid and accurate detection of microorganisms in the acute-care setting. Over the past years the application of molecular diagnostics has started a revolution in the diagnosis of infectious diseases. In particular, the advent of nucleic acid based assays has revolutionised the laboratory diagnosis of viral and bacterial infections of the CNS.

A flow chart of the sequential laboratory steps to be followed for CNS infection diagnosis is shown in Figure 1.

The most developed molecular method is the polymerase chain reaction (PCR), which is a technique widely used in microbiology, since it can amplify viral and bacterial nucleic acid from cerebrospinal fluid (CSF) and is now considered the gold standard for the diagnosis of CNS diseases. In fact CSF, which can be taken in a less invasive way than brain biopsy, is abnormal in $90 \%$ of cases of viral encephalitis, but routine CSF tests rarely lead to identification of specific aetiologic agents. Another important innovation is real-time PCR, which is used to evaluate the viral and bacterial genome copy number. Viral load may be considered as a marker for the severity of disease or may help to predict outcome, although it remains to be determined whether this is true for all infections [1].

e-mail: pasquale.ferrante@unimi.it.

Conflict of Interest statement The Authors declare that they have no conflict of interest related to the publication of this manuscript 
All the explained molecular methods have both higher sensitivity and specificity compared to conventional microbiological methods. Moreover, they are time-saving and can be applied to identification of organisms that cannot be grown in vitro. Beside the advantages, the use of molecular methods also has some limitations. The principal shortcomings in applying PCR assays to the clinical setting include false-positive results from DNA contamination; the potential for false-negative results; the necessity to run external and internal quality control; and finally, the relatively high cost. For all these reasons a limited number of assays have been approved by the US Food and Drug Administration (FDA) and many still have to achieve universal acceptance in clinical practice. Up to now, PCR and related methods have been widely available and been of proven efficacy in the diagnosis of herpes simplex virus (HSV), which is the most common cause of acute sporadic focal encephalitis. A recent review estimated that the sensitivity of CSF PCR for the diagnosis of HSV is $96 \%$ and that the specificity is $99 \%$ [2]. About the same percentages characterise molecular methods used to detect enteroviruses, Epstein-Barr virus and human cytomegalovirus [3] in the CSF, whereas sensitivity and specificity rates decrease for the VZV, HHV6, HIV and rabies viruses.

The experience of our group consists of the optimisation of many molecular tests to detect viruses in the CSF. In particular, our studies have been focusing for more than ten years on the analysis of human polyomavirus JCV, the aetiological agent of PML, a fatal demyelinating disease of the white matter of the CNS [4]. JCV is a slow-growing virus (up to five weeks), so that a diagnostic test different from culturing is needed. Previously, the virus was searched by means of a nested PCR that had 92\% sensitivity, with limit of detection of 1000 virus copies/ $\mathrm{ml}$, and $100 \%$ specificity [5]. However, after the introduction of HAART therapy, the PCR technique showed a strong decrease in the sensitivity, because of the lower amount of virus in the CSF, due to the restoration of the immune system. The advent of real-time PCR contributed to improving a new diagnostic specific method, because of its very high sensitivity (detection limit: 500 copies $/ \mathrm{ml}$ ). Our preliminary results demonstrated that JCV viral load in the CSF samples, collected both at enrolment and during follow up, from PML patients, could be associated with the clinical course of the disease. JCV viral load was in fact significantly higher $(p<0.01)$ in 10 CSF samples of fast progressing PML patients (5.51 log copies/ml) than in 12 CSF samples of slow progressing PML patients (4.14 log copies $/ \mathrm{ml})$. Thus we can conclude that PCR technologies have revolutionised the laboratory diagnostics of leukoencephalopathies and both physicians and patients can benefit from molecular methods applied in neuroinfectology.

\section{References}

1. Aberle SW, Puchhammer-Stockl E. Diagnosis of herpesvirus infections of the central nervous system. J Clin Virol 2002;25:S79-S85. [PubMed: 12091085]

2. Tebas P, Nease RF, Storch GA. Use of the PCR in the diagnosis of herpes simplex encephalitis: a decision analysis model. Am J Med 1998;105:287-295. [PubMed: 9809689]

3. Bestetti A, Pienotti C, Terreni M, et al. Comparison of three nucleic acid amplification assays of cerebrospinal fluid for diagnosis of cytomegalovirus encephalitis. J Clin Microbiol 2001;39:11481151. [PubMed: 11230445]

4. Walker, DL.; Padgett, BL. The epidemiology of human polyomaviruses. In: Sever, JL.; Madden, D., editors. Polyomaviruses and human neurological disease. Alan R. Liss; New York: 1983. p. 99-106.

5. Ferrante $\mathrm{P}$, Caldarelli-Stefano R, Omodeo-Zorini E, et al. Comprehensive investigation of the presence of JC virus in AIDS patients with and without progressive multifocal leukoencephalopathy. J Med Virol 1997;52:235-242. [PubMed: 9210030] 


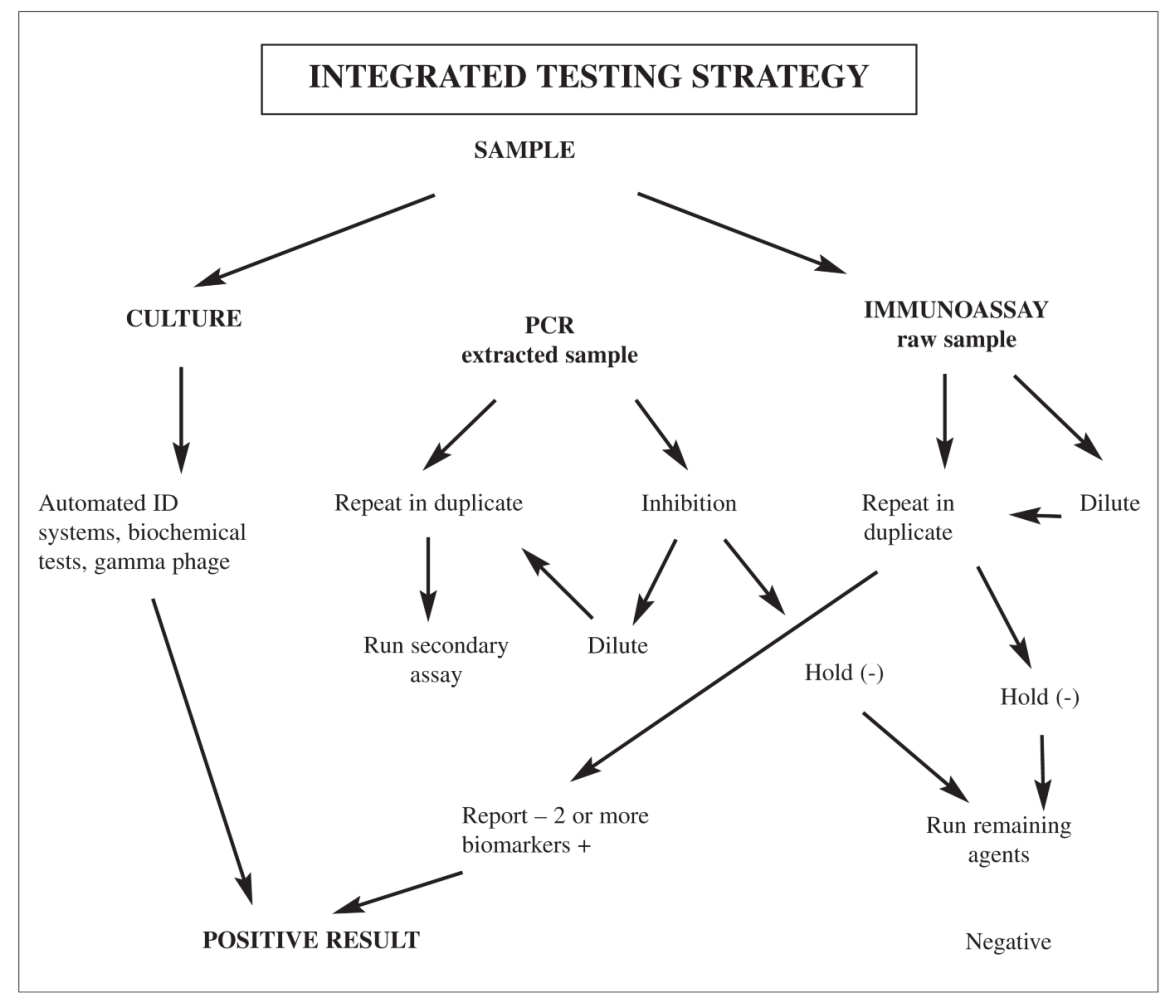

Fig. 1.

Flow chart of the sequential laboratory steps to be followed for CNS infection diagnosis 